\title{
Traducción y adaptación cultural del Yale-Brown Obsessive Compulsive Scale (Y-BOCS) para trastornos obsesivos compulsivos
}

\section{Spanish translation and cultural adaptation of Yale- Brown Obsessive Compulsive Scale (Y-BOCS)}

Correspondencia

Giuliana Angelica Yacila

Huaman

giuliana.yacila@gmail.com

Recibido: 09/08/2016

Aprobado: 17/08/2016

Citar como: Yacila GA, Cook-del Aguila L, Sanchez-Castro AE, Reyes-Bossio M, Tejada $R A$. Traducción y adaptación cultural del Yale-Brown Obsessive Compulsive Scale (Y-BOCS) para trastornos obsesivos compulsivos. Acta Med Peru. 2016;33(3):253-5

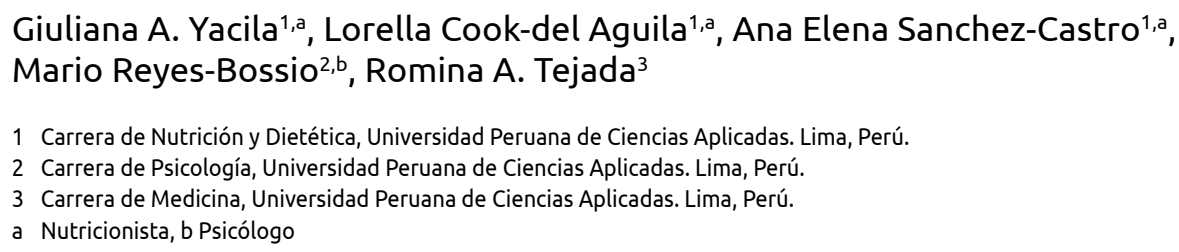
Mario Reyes-Bossio ${ }^{2, b}$, Romina A. Tejada ${ }^{3}$

1 Carrera de Nutrición y Dietética, Universidad Peruana de Ciencias Aplicadas. Lima, Perú.

2 Carrera de Psicología, Universidad Peruana de Ciencias Aplicadas. Lima, Perú.

3 Carrera de Medicina, Universidad Peruana de Ciencias Aplicadas. Lima, Perú.

a Nutricionista, b Psicólogo

\section{Sr. Editor:}

La quinta edición del Manual Estadístico y Diagnóstico de Trastornos Mentales describe a los trastornos obsesivos-compulsivos (TOC) como la preocupación obsesiva constante -real o no-y el comportamiento o conducta compulsiva repetitiva, para neutralizar aquellos pensamientos recurrentes y persistentes [1]. Estas personas intentan ignorar o desaparecer los pensamientos, impulsos o imágenes mentales generando ansiedad o estrés en un individuo. Estos pensamientos o actos afectan al desenvolvimiento social y laboral porque consume tiempo dedicado a esta obsesión. La Organización Mundial de la Salud ha considerado este trastorno como una de las diez enfermedades más incapacitantes ya que la persona puede llegar a evitar el contacto social o personal ${ }^{[2]}$. Este trastorno afecta más a varones en edades tempranas, pero, al llegar a la adultez, las mujeres entre 18-44 años son quienes tienden a presentar más un TOC ${ }^{[3]}$.

Se ha determinado que la prevalencia oscila entre 1,5 a 3,5\% en la población general ${ }^{[4]}$, y entre el 10 y $63 \%$ de personas con TOC presentan ideas suicidas. Para un manejo adecuado, es necesario hacer una detección precoz, y para ello se requiere tener instrumentos válidos, de fácil aplicación y adaptados al contexto cultural de cada país ${ }^{[5]}$.

El cuestionario autoaplicado de Yale-Brown Obsessive Compulsive Scale (Y-BOCS) fue adaptado por Steketee para medir la presencia de unTOC [6]. EI Y-BOCS está compuesto por 10 ítems: 5 relacionado a obsesiones y los otros 5, a compulsiones, teniendo una opción de respuesta de o a 4 (desde no presentar un síntoma hasta presentar síntomas extremos). La clasificación de diagnóstico se basa en los puntos de corte donde o a 7 representa "sin manifestaciones clínicas", 8 a 15, "leve", 16 a 23, "moderado", 24 a 31 como "severo" y 32 a 40 como "extremo". Este cuestionario presenta un alfa de Cronbach de 0,78 para personas con TOC y de 0,89 para personas sin manifestaciones clínicas ${ }^{[6]}$. El cuestionario completo original de Goodman ha sido traducido y validado al español para el uso pediátrico ${ }^{[7]}$. Sin embargo, no se han encontrado traducciones al español del cuestionario autoaplicado para adultos. Este cuestionario tiene cinco subescalas: tiempo dedicado, interferencia, angustia, resistencia y control.

Se realizó la traducción del cuestionario Y-BOCS junto con la del cuestionario Muscle Appearance Satisfaction Scale ${ }^{[8]}$ para ser utilizados en un trabajo posterior sobre dismorfia muscular. Se generó una versión en español a partir de la traducción delY-BOCS de inglés a castellano por un traductor oficial y de un profesional de la salud, que fue luego sintetizada por el equipo de investigadores. Esta versión fue evaluada por diez expertos con diferente formación profesional siguiendo la metodología Delphi 
Tabla 1. Versión al español del cuestionario Yale-Brown Obsessive Compulsive Scale (Y-BOCS).

\section{Yale-Brown Obsessive Compulsive Scale (Y-BOCS) en español}

1. ¿Cuánto de tu tiempo lo ocupas en pensamientos persistentes sobre un defecto o falla en tu apariencia?
a) Ninguno
b) Menos de 1 hora al día
c) 1 a 3 horas al día
d) 3 a 8 horas al día
e) Más de 8 horas al día

3. ¿Cuánta angustia te causan los pensamientos persistentes sobre tus defectos corporales?
a) Ninguna
b) Ligera, no muy perturbadores
c) Moderada, perturban, pero es manejable
d) Severa, muy perturbadores
e) Extrema, angustia incapacitante

5. ¿Cuánto control tienes sobre los pensamientos persistentes sobre tus defectos corporales?

a) Control completo

b) Mucho control, usualmente capaz de detener o desviar estos pensamientos persistentes con algo de esfuerzo y concentración

c) Control moderado, algunas veces es capaz de detener o desviar estos pensamientos persistentes.

d) Poco control, muy rara vez tiene éxito en detener los pensamientos persistentes.

e) Ningún control, rara vez capaz de desviar aún por un momento la atención

7. ¿Cuánto interfieren las conductas repetitivas relacionadas a tus defectos corporales con tus funciones (roles) sociales, de trabajo u otros?
a) Ninguna
b) Pequeña interferencia, pero no hay impedimento.
c) Hay interferencia definitiva pero manejable
d) Impedimento substancial
e) Interferencia extrema, incapacitante

9. ¿Cuánto esfuerzo realizas para resistir estas conductas repetitivas?
a) No necesita resistir
b) Trata de resistir la mayor parte del tiempo
c) Hace algún esfuerzo de resistir
d) Cede a todas esas conductas repetitivas
e) Cede completa y voluntariamente a todas las conductas repetitivas

2. ¿Cuánto interfieren los pensamientos persistentes de tus defectos corporales con tus funciones (roles) sociales, de trabajo $\mathrm{u}$ otros?
a) Ninguno
b) Pequeña interferencia, pero no hay impedimento.
c) Hay interferencia definitiva pero manejable
d) Impedimento substancial
e) Interferencia extrema, incapacitante

4. ¿Cuánto te esfuerzas para resistirte a estos pensamientos persistentes?
a) No necesita resistir
b) Trata de resistir la mayor parte del tiempo
c) Hace algún esfuerzo de resistir
d) Cede a todos esos pensamientos persistentes
e) Cede completa y voluntariamente a todos los pensamientos persistentes

6. ¿Cuánto tiempo le dedicas a realizar conductas repetitivas relacionadas a tu defecto o falla en tu apariencia?
a) Ninguno
b) Menos de 1 hora al día
c) 1 - 3 horas al día
d) 3 - 8 horas al día
e) Más de 8 horas al día

8. ¿Cuán ansioso te pondrías si te impidieran realizar tus conductas repetitivas relacionadas a tus defectos corporales?
a) Sin ansiedad
b) Sólo ligeramente ansioso
c) Poca ansiedad, pero manejable
d) Ansiedad prominente y perturbadora
e) Extrema, ansiedad incapacitante

10. ¿Cuánto control tienes sobre realizar tus conductas repetitivas relacionadas a tus defectos corporales?
a) Control completo
b) Mucho control, usualmente capaz de detener o desviar estas conductas repetitivas con algo de esfuerzo y concentración
c) Control moderado, algunas veces es capaz de detener o desviar estas conductas repetitivas
d) Poco control, muy rara vez tiene éxito en detener las conductas repetitivas
e) Ningún control, rara vez capaz de desviar aún por un momento la atención 
para evaluar la adaptación cultural de esta versión [9], entre las principales modificaciones en esta fase fue el cambio de la frase "pensamientos persistentes" en los primeros cinco enunciados para evitar inducir la respuesta, y se cambió "actividades" por "conductas repetitivas", para indicar la frecuencia constante de estas actividades (Tabla 1). Se obtuvo un coeficiente de validez V de $0,9^{[10]}$.

Esta versión fue evaluada en una muestra de 618 estudiantes jóvenes entre 18 y 30 años en una universidad privada de Lima, Perú. Se calculó la consistencia interna, presentando un alfa de Cronbach global de 0,89, igual que la versión adaptada delY-BOCS en pacientes $\sin$ manifestaciones clínicas ${ }^{[6]}$. Las dos subescalas tuvieron un alfa de Cronbach de 0,83 mientras que la versión de Steketee presenta 0,85 y 0,84 para la subescala de obsesivo y compulsivo, respectivamente. Los grupos poblacionales tienen aspectos similares, ambos siendo estudiantes universitarios, pero de diferente género. Tanto la versión de Steketee y la realizada en este estudio son versiones autoaplicadas con una consistencia interna fiable para ser utilizadas en una población general. Futuros estudios deben evaluar si la estructura factorial de los dominios es similar al original, y si la estabilidad de la medición es buena aplicando un test-retest. Así como evaluarlo en personas con diagnóstico clínico de TOC para determinar la sensibilidad de los puntos de corte establecidos.

En conclusión, se obtuvo una versión al español del Y-BOCS, adaptada culturalmente al contexto peruano, la cual tiene una buena consistencia interna.

Fuente de financiamiento: Los autores declaran no haber recibido ninguna financiación para la realización de este trabajo.

Conflicto de intereses: Los autores declaran no tener conflicto de intereses con la publicación de este artículo.

\section{REFERENCIAS BIBLIOGRÁFICAS}

1. American Psychiatric Association. Diagnostic and statistical manual

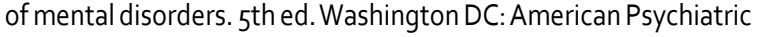
Association; 2013.

2. Rosario-Campos MC, Miguel EC, Quatrano $S$, Chacon P, Ferrao $Y$, Findley $D$, et al. The Dimensional Yale-Brown ObsessiveCompulsive Scale (DY-BOCS): an instrument for assessing obsessive-compulsive symptom dimensions. Mol Psychiatry. 2006;11(5):495-504.

3. Goodman WK, Grice DE, Lapidus KA, Coffey BJ. Obsessivecompulsive disorder. Psychiatr Clin North Am. 2014;37(3):257-67.

4. Angelakis I, Gooding P, Tarrier N, Panagioti M. Suicidality in obsessive compulsive disorder (OCD): A systematic review and meta-analysis. Clin Psychol Rev. 2015i39:1-15. doi: 10.1016/j. cpr.2015.03.002.

5. Tortella-Feliua M, Bañosa RM, Barrantesa N, Botella C, FernándezAranda F, García-Campayo J, et al. Retos de la investigación psicológica en salud mental. Clínica y Salud. 2016;27(1):37-43.

6. Steketee G, Frost R, Bogart K. The Yale-Brown Obsessive Compulsive Scale: interview versus self-report. Behav Res Ther. 1996;34(8):675-84.

7. Sal y Rosas H.J, Vega-Dienstmaier J.M, Mazzotti G, Vidal H, Guimas $B$, Adrianzén C, Vivar R. Validación de una versión en español de la Escala Yale-Brown para el Trastorno Obsesivo-Compulsivo. Actas Esp Psiquiatr. 2002;30(1):30-5.

8. Cook-del Aguila L, Sanchez-Castro AE, Yacila GA, Reyes Bossio MA, Mayta-Tristán P. Adaptación cultural de la versión peruana de la Muscle Appearance Satisfaction Scale (MASS) para dismorfia muscular. Rev Colomb Psiquiatr. 2016. In press. http://dx.doi. org/10.1016/j.rcp.2016.04.003.

9. Escobar-Perez J, Cuervo-MartinezA.Validez de contenido y juicio de expertos: una aproximación a su utilización. Avances en Medición. 2008;6(1):27-36.

10. Escurra LM. Cuantificación de la validez de contenido por criterio de jueces. Rev Psicol. 1988;6(1-2):103-11.

\section{Las ediciones anteriores de Acta Médica Peruana están disponibles en:}

\section{www.scielo.org.pe}

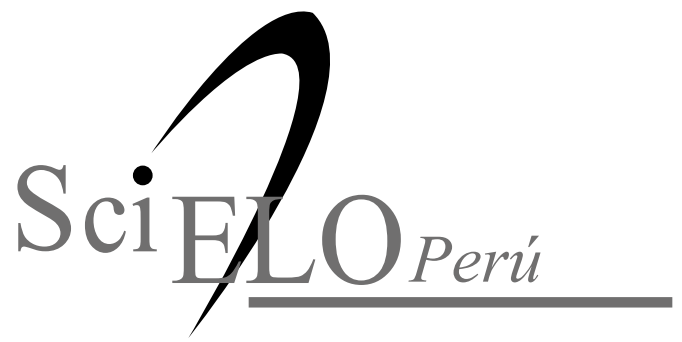

